\begin{tabular}{|l|l|l||}
\hline \multicolumn{2}{|c|}{ PublisherInfo } \\
\hline \hline PublisherName & $:$ & BioMed Central \\
\hline \hline PublisherLocation & $:$ & London \\
\hline \hline PublisherImprintName & $:$ & BioMed Central \\
\hline \hline
\end{tabular}

\title{
Pathologic findings from the NSABP B-17 protocol: 8 year update
}

\begin{tabular}{|l|l|l||}
\hline \multicolumn{2}{|c||}{ ArticleInfo } \\
\hline \hline ArticleID & $:$ & 3623 \\
\hline \hline ArticleDOI & $:$ & $10.1186 /$ bcr-1999-66601 \\
\hline \hline ArticleCitationID & $:$ & 66601 \\
\hline \hline ArticleSequenceNumber & $:$ & 43 \\
\hline \hline ArticleCategory & $:$ & Paper Report \\
\hline \hline ArticleFirstPage & $:$ & 1 \\
\hline \hline ArticleLastPage & $:$ & 4 \\
\hline \hline & & RegistrationDate : 1999-8-9 \\
ArticleHistory & $:$ & OnlineDate \\
\hline \hline ArticleCopyright & $:$ & Current Science Ltd1999-8-9 \\
\hline \hline ArticleGrants & $:$ & \\
\hline \hline ArticleContext & $:$ & 1305811 \\
\hline \hline
\end{tabular}




\section{Keywords}

breast, intraductal carcinoma, ductal carcinoma in situ, pathology, treatment

\section{Introduction}

The National Surgical Adjuvant Breast Project (NSABP) protocol B-17 trial was set up to determine whether the addition of radiotherapy after local excision was beneficial in maintaining local control and extending survival in patients with ductal carcinoma in situ (DCIS). Its findings at five years showed a significant reduction in ipsilateral breast tumor recurrence in those patients who had received radiotherapy as opposed to those who had not (16.4\% versus 7.0\%). Pathological analysis to identify discriminants predictive of local recurrence found that only the presence of moderate to marked comedo necrosis and uncertain or involved margins of resection were found to be independent high risk factors. The present report updates the previous findings, with clinical follow-up now being available for eight years.

\section{Aims}

To identify pathological features of DCIS which may be important in predicting ipsilateral tumor recurrence, other events, and selection of breast irradiation following lumpectomy.

\section{Comments}

The NSABP B-17 trial represents the largest group of prospectively evaluated, randomized patients undergoing uniform treatment and thus provides valuable information for the management of DCIS. The finding that comedo necrosis is a significant predictor of disease recurrence strengthens the case for morphological DCIS classifications based on the presence or absence of necrosis. However, a recent study by 23 European pathologists showed a surprising inability to identify comedo DCIS consistently and thus casts some doubt on the ability of pathologists to accurately score the presence and extent of necrosis. A rather surprising finding of the present study was that margin status had only a slight or borderline influence on the incidence of local recurrence. DCIS is usually a unicentric disease and thus should be cured if adequate excision is possible. This unexpected finding might be explained by the 
definitions for margin status used and by the differences in histopathology reporting now and in the 1980s, when accrual of patients for the study began. Overall, the study reiterates its earlier message and confirms the usefulness of radiotherapy in the management of DCIS.

\section{Methods}

The study included 814 patients, 24 in addition to that previously reported. On 623 (77\% of the entire B-17 cohort) central pathological review was performed and of these 303 were treated by lumpectomy and 320 by lumpectomy with subsequent radiotherapy (10 Gray per week for five weeks). The nine pathological features assessed were comedo necrosis, histologic type, excision margins, lymphoid infiltrate, nuclear grade, focality, cancerization, stroma and tumor size. The Van Nuys pathological grade was also calculated; however, the data were not suitable to calculate the Van Nuys prognostic index.

\section{Results}

The cumulative frequency for local recurrence was 137 (22\%) for all 623 patients; 94 (31\%) occurred in those treated by local excision only and $43(13 \%)$ in those who received additional radiotherapy. The relative risk for radiotherapy was 0.39 (95\% Confidence Interval (CI), 0.27-0.56); representing a 61\% relative reduction. The recurrences occurred within 2 to 123 months (median 36 months) after surgery and presented earlier in those treated by local excision alone. The average annual hazard rates for local recurrence were lower for all nine pathological criteria in patients who had also received radiotherapy. However, many of the pathological features are interrelated and thus, when they were examined jointly for prognostic significance, only moderate-to-marked and absent-to-slight comedo necrosis were found to be independent high and low risk predictors respectively. When the Van Nuys pathological classification was used, the lesions in groups 2 and 3 represented high risk categories for local recurrence in patients treated with local excision alone, whereas all three groups showed a benefit from the addition of radiotherapy.Local recurrence of breast tumor was the most frequent event. Other events included contralateral breast carcinoma (4.3\%) and second primary cancers $(3.2 \%)$. Of the 137 local breast recurrences $61 \%$ were "pure" DCIS and in $90 \%$ of these the DCIS was morphologically similar to the index lesion. The overall mortality due to breast cancer for the cohort was only $1.6 \%$ at eight years.

\section{Discussion}

This study has shown that, in patients with DCIS, the extent of comedo necrosis enables high and low risks for local recurrence following lumpectomy to be distinguished. Margin status, unlike the results reported in the previous report, showed only a slight or borderline influence on the incidence of local 
recurrence at eight years. When the data were analyzed using the Van Nuys pathological classification all three groups exhibited a significant reduction of local recurrence from radiotherapy. The decision not to use radiotherapy in the low risk group would appear to depend, therefore, on clinical considerations and on the input of informed patients, rather than being standard practice.

\section{Additional information}

See accompanying editorial, Morrow M: Understanding ductal carcinoma in situ. A step in the right direction. Cancer 1999; 86: 375-377.

\section{References}

1. Fisher ER, Dignam J, Tan-Chiu E, Costantino J, Fisher B, Paik S, Wolmark N: Pathologic findings from the national surgical adjuvant breast project (NSABP) eight year update of protocol B-17. Cancer. 1999, 86: 429-438. 